\title{
Beste Lösungen für implantatgetragenen Zahnersatz
}

VITA IMPLANT SOLUTIONS (IS) sind neue CAD/CAM-Rohlinge für implantatgetragenen Zahnersatz aus Hybridkeramik, Glaskeramik und Komposit, die die VITA Zahnfabrik zur IDS 2015 erstmals vorstellte. Die Rohlinge verfügen über eine integrierte Schnittstelle (Schraubenkanal inkl. Verdrehsicherung) zur Klebe-/Titanbasis (z. B. TiBase). Damit sind die Rohlinge zu den Implantsystemen vieler Hersteller kompatibel. Die IS-Rohlinge werden als VITA ENAMIC IS, VITA SUPRINITY IS und VITA CAD-Temp IS angeboten. Für die definitive Versorgung empfehlen sich VITA ENAMIC IS-Rohlinge. Die Hybridkeramik kann dank dentinähnlicher Elastizität einwirkende Kaukräfte absorbieren. Die Herstellung der VITA ENAMIC IS Suprakonstruktionen ist darüber hinaus sehr zeitund kostensparend umsetzbar, da das Material nicht nur enorm präzise, sondern auch schnell CAD/CAM-technisch verar- beitbar ist. Mit VITA SUPRINITY IS wurde auch die hochfeste, zirkondioxidverstärkte Glaskeramik für definitive Suprakonstruktionen präsentiert.

Die Rohlinge aus beiden Materialvarianten werden in 2 Geometrien und Transluzenzstufen angeboten. Damit lassen sich sowohl 2-teilige Versorgungen, bestehend aus Mesostruktur und Krone, als auch 1-teilige Versorgungen in Form von monolithischen Abutment-Kronen herstellen. Die 1-teilige Lösung empfiehlt sich primär im Seitenzahnbereich und die 2-teilige Lösung für implantatgetragene Kronenrekonstruktionen im Frontzahnbereich. Die VITA IMPLANT SOLUTIONS werden mit den VITA CAD-Temp IS-Rohlingen aus Komposit zur temporären Versorgung ab-

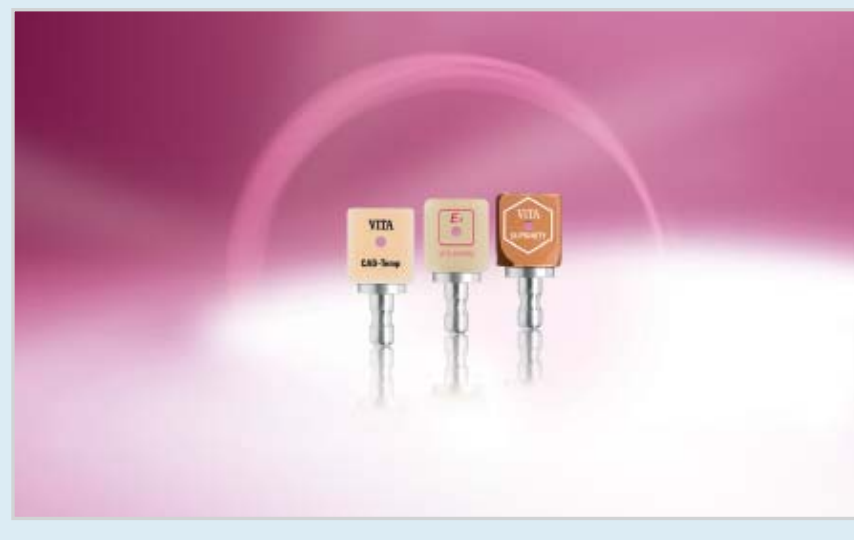

gerundet. Diese dienen neben der provisorischen Versorgung vor allem der individuellen Gestaltung des Emergenzprofils.

Nach einer Pressemitteilung der VITA Zahnfabrik H. Rauter GmbH \& Co. KG, Bad Säckingen 